\title{
Assessing Alternative Technologies for the Cost-Effective Computation of Derivatives
}

\author{
Roy S. Freedman \\ Inductive Solutions, Inc. \\ Roy@Inductive.com
}

\author{
Rinaldo DiGiorgio \\ Sun Microsystems \\ Rinaldo.DiGiorgio@East.Sun.com
}

\begin{abstract}
One should not separate the method of computing the expected present value of a derivative from its ultimate computing topology. In the following sections, we discuss the cost-benefit issues involved with implementing several methods for computing derivative statistics on alternate computing topologies. We show how the choice of topology impacts the computing time for a particular example of a time consuming derivative valuation. We conclude by showing how all these factors can be represented as a case-based expert system, which can be used to help an organization assess its computing alternatives.
\end{abstract}

\section{Introduction}

There are several ways of incorporating additional computing power to speed up the computation of derivatives, and the "obvious" answer of "getting a faster computer" may not be obvious, or may even be "obviously wrong." For example:

"We have alot of programmers who write $\mathrm{C}$ applications. We have alot of Unix workstations, but most are efficiently used all day and all night. Our derivative evaluation application is based on Monte Carlo methods, and we need to improve the accuracy without sacrificing time."

"We need to evaluate our very large portfolio in almost real time. We already have a supercomputer but we could use 2 more. Should we buy another million-dollar parallel processor? P.S. We have alot of idle workstations."

"We run a lattice-type American-style valuation application each day on my entire inventory. We can do one evaluation each day. We keep getting more clients. Should I go back to a Black-Scholes formula? My application runs on a PC and I do not understand parallel computation. We have no programmers on staff." 
The alternative computing topologies considered here are (listed in order of increasing cost):

1. Workstations

2. Faster Workstations

3. Supercomputers

4. Networked ("Clustered") Systems, that could contain both workstations, supercomputers, or both.

Their general characteristics are summarized in Figure 1.

\begin{tabular}{|ccccc|} 
& $\begin{array}{c}\text { Number of } \\
\text { Processors }\end{array}$ & $\begin{array}{c}\text { Speed in } \\
\text { MFLOPS }\end{array}$ & $\begin{array}{c}\text { Memory in } \\
\text { MBytes }\end{array}$ & $\begin{array}{c}\text { Cost } \\
\$ \mathrm{~K}\end{array}$ \\
\cline { 2 - 5 } Workstation & 1 & $1-25$ & 32 & $<\$ 10 \mathrm{~K}$ \\
Faster Workstation & $1-4$ & $>25$ & 64 & $>10 \mathrm{~K} \$$ \\
Supercomputer & $>1$ & 2000 & $>128$ & $>1000 \$ \mathrm{~K}$ \\
Cluster & $>1$ & $>2000$ & $>128$ & $>\$ 40 \mathrm{~K}$ \\
\hline
\end{tabular}

Figure 1. Characteristics of Alternate Computing Topologies

The essential idea of this paper is that one should not separate the method of computing the expected value of a derivative from its ultimate computing topology. Different topologies may be more cost-effective than other topologies.This is a point also made in [1], even though their evaluation was basically concerned with showing the computing potential of the cluster topology, not its cost-benefit tradeoffs with respect to an organization's requirements.

In the following sections, we discuss the cost-benefit issues involved with implementing different methods for evaluating a derivative on alternate computing topologies. We first survey the basic algorithmic approaches in computing derivatives and group them into three basic methods. We then show how the topology impacts the computing time for a particular example of a time consuming derivative valuation. We conclude by showing how all these factors can be represented as a case-based expert system, which can be used to help an organization assess its computing alternatives.

\section{Algorithm Tradeoffs in Computing Derivatives}

We are concerned with the computational problem of deriving the expected value and other statistics of a derivative security $f$ at time $T_{0}$. When the underlying security $S$ and derivative security $\mathrm{f}$ are modeled as stochastic processes, the problem can be solved by reformulating it as a boundary-value problem: if it is known that the derivative pays out $\mathrm{f}_{\mathrm{T}}$ at time $\mathrm{T}$, we just compute its value backwards from the risk adjusted random price movements of the underlying from $t=T$ to $t=T_{0}$. The present value of $f$ is just its expected discounted value in a risk-neutral world

$$
\text { Expected Present Value } \left.=E\left[e^{-r(T-T o}\right)_{f_{T}}\right]
$$


Here, $r$ is the average instantaneous risk-free interest rate between $t=T_{0}$ and $t=T$. When the underlying $S$ follows an Ito process, and if the derivative is some differentiable function of $S$ and $t$, $\mathrm{f}=\mathrm{f}(\mathrm{S}, \mathrm{t})$, then by Ito's Lemma, $\mathrm{f}$ also follows an Ito process:

$$
\begin{aligned}
\mathrm{dS} & =\mu(\mathrm{t}, \mathrm{S}) \mathrm{dt}+\sigma(\mathrm{t}, \mathrm{S}) \mathrm{dz} \\
\mathrm{df} & =(\partial \mathrm{f} / \partial \mathrm{S}) \mathrm{dS}+\left[\partial \mathrm{f} / \partial \mathrm{t}+(1 / 2) \sigma^{2}(\mathrm{t}, \mathrm{S})\left(\partial^{2} \mathrm{f} / \partial \mathrm{S}^{2}\right)\right] \mathrm{dt} \\
& =\left[\mu(\mathrm{t}, \mathrm{S})(\partial \mathrm{f} / \partial \mathrm{S})+\partial \mathrm{f} / \partial \mathrm{t}+(1 / 2) \sigma^{2}(\mathrm{t}, \mathrm{S})\left(\partial^{2} \mathrm{f} / \partial \mathrm{S}^{2}\right)\right] \mathrm{dt}+\sigma(\mathrm{t}, \mathrm{S})(\partial \mathrm{f} / \partial \mathrm{S}) \mathrm{dz}
\end{aligned}
$$

and $\mathrm{f}$ satisfies the Fokker-Plank forward diffusion equation

$$
\begin{gathered}
\partial \mathrm{f} / \partial \mathrm{t}=(1 / 2)\left(\partial^{2} / \partial \mathrm{S}^{2}\right)\left[\sigma^{2}(\mathrm{t}, \mathrm{S}) \mathrm{f}\right]-(\partial / \partial \mathrm{S})[\mu(\mathrm{t}, \mathrm{S}) \mathrm{f}] \\
\text { given initial condition } \mathrm{S}\left(\mathrm{T}_{0}\right)=\mathrm{S}_{0}
\end{gathered}
$$

Here $S(t)$ is the probability distribution of the price of the underlying at time $t, \mu(t, S)$ and $\sigma(t, S)$ are the instantaneous drift and standard deviation rates, and dz is a Wiener Process that corresponds to Brownian motion. Note that if we know the probability distributions for $\mathrm{S}(\mathrm{t})$, and if we are given boundary conditions for $\mathrm{f}$ (which define the derivative), then we can solve (2FP) and derive the probability distribution for $f$, so that the expected present value of $f$ can be computed from Equation (1).

The above equations are valid for all derivative securities with $\mathrm{S}$ as the underlying stochastic variable [4]. A vector form of Equation (2S) and (2f) is valid if $\mathrm{S}$ depends on other Ito processes (for example, if $\mu$ or $\sigma$ are Ito processes). Here, the correlations of the underlying processes are additional factors in the dt term in Equation (2f).

Simplifications can be made: if the interest rate $r$ is known to be constant, then it can be shown that the Ito process for $[S(\partial f / \partial S)-f]$ does not depend on $d z$ - this "continuous" hedge is "riskless." Hence, in this case, f satisfies the Black-Scholes partial differential equation

$\partial \mathrm{f} / \partial \mathrm{t}=\mathrm{rf}-(1 / 2) \mathrm{S}^{2} \sigma^{2}(\mathrm{t}, \mathrm{S})\left(\partial^{2} \mathrm{f} / \partial \mathrm{S}^{2}\right)-\mathrm{rS}(\partial \mathrm{f} / \partial \mathrm{S})$

Equation (2BS) can be solved if $\mathrm{S}(\mathrm{t})$ is known and the boundary conditions that define the derivative $\mathrm{f}$ are provided. For example, a boundary condition for a European call option is

When $\mathrm{t}=\mathrm{t}, \mathrm{f}(\mathrm{T})=\mathrm{f}_{\mathrm{T}}=\max \left(\mathrm{S}_{\mathrm{T}}-\mathrm{X}, 0\right)$

In practice, in all but the simplest cases, the price movements of $\mathrm{S}$ and f follow stochastic processes that involve substantial amounts of computation. There are three general methods that have different computational consequences for computing European-style (the holder has no decisions to make during its life) and American-style (the holder has decisions to make during its life) derivatives: 
Method 1. Analytic Approximation for Constant Parameters. If the derivative is a Europeanstyle derivative, and the Ito process in Equations (2S), (2f), and (2BS) has constant $\mu(\mathrm{t}, \mathrm{S})=\mu$, constant $\sigma(\mathrm{t}, \mathrm{S})=\sigma$, and constant interest rate, then computationally nice expressions exist for the derivative security - the famous formulas derived by Black and Scholes. Analytic expressions also exist for approximating the values of American-style derivatives. In Method 1, the time required to compute the expected value of $\mathrm{f}$ is proportional to a constant factor $\mathrm{G}$ - the time required to evaluate the formula. In general, $\mathrm{G}$ depends on the efficiency of computation of special functions (like the normal distribution).

Method 2. Recombining Lattice-Type Computations. If the Ito process in Equations (2S), (2f), and (2BS) has constant $\mu(\mathrm{t}, \mathrm{S})=\mu$, constant $\sigma(\mathrm{t}, \mathrm{S})=\sigma$, and constant interest rate, then the valuation of a European- or American-style derivative is usually computed by simulating the updown price movements in a recombining binomial lattice. (The lattice is a discrete form of Equation (2S-2f), and is also related to a discrete form of (2FP) and (2BS)). In this method, the time required to compute the value of a derivative depends on the number of time units $\mathrm{N}$, where $\mathrm{N}=\left(\mathrm{T}-\mathrm{T}_{0}\right) / \Delta \mathrm{t}$, and $\Delta \mathrm{t}$ is the smallest unit of time considered in the computation. In this method, a sequence of up movements followed by down movements are valued the same as the down movements followed by the up movements. At any given point in time $\mathrm{T}_{0}+\mathrm{i} \Delta \mathrm{t}$, the price of the underlying may increase or decrease by an amount $u$ and $d$ with probability $p$ and (1-p) respectively. Hence, at time $\mathrm{T}_{0}+\mathrm{i} \Delta \mathrm{t}$, the price of the underlying may be any of a set of $\mathrm{i}+1$ values: $\mathrm{Su}_{\mathrm{u}} \mathrm{j}_{\mathrm{d}}^{\mathrm{i}-\mathrm{j} ;} \mathrm{i}=0, . . \mathrm{N} ; \mathrm{j}=0, . ., \mathrm{i}$. A recombining binomial lattice must compute and store a total of $(\mathrm{N}+1)(\mathrm{N}+2) / 2$ prices for the underlying and derivative. For $\mathrm{N}=500$, this requires approximately $10^{5}$ computations, and represents much greater computational overhead than Method 1. This method may require several orders of magnitude of computation than Method 1.

Method 3. Non-Recombining Simulation. If $\mathrm{f}$ is a European-style derivative, and the Ito process in Equations (2S), (2f), and (2FP) has non-constant $\mu(\mathrm{t}, \mathrm{S})$, non-constant $\sigma(\mathrm{t}, \mathrm{S})$, and possibly non-constant interest rate, then Method 2 may not work because the up values and down values of a price movement may not combine: a sequence of up movements followed by down movements are not valued the same as the down movements followed by the up movements. Consequently, in evaluating the possible price of $\mathrm{S}$, after $\mathrm{N}$ time increments there are $2^{\mathrm{N}+1}$ possible prices (none are recombined as in Method 2; if recombining is allowed, there are only $(\mathrm{N}+1)(\mathrm{N}+2) / 2$ prices). In Method 3, where recombining is not possible, all $2^{\mathrm{N}+1}$ possible prices must be generated to get the "complete" distribution for the expected value in Equation (1). Pragmatically, this is impossible, since for $n=500$, this is approximately $10^{150}$ prices. The alternative here is to create a representative random "Monte Carlo sample" of $f$ so that the expectation in Equation (1) can be computed directly from the random sample of prices, and not from the complete set of prices. In Method 3, the time required to compute the value of a derivative depends on the number of discrete time units $\mathrm{N}$ and the number of Monte Carlo samples $\mathrm{M}$ generated for $\mathrm{f}$. Accuracy in the evaluation of $\mathrm{f}$ is a statistical problem relating to the standard error of the estimate of the sample mean. Since it is known that the standard error in computing an expectation is proportional to $\mathrm{M}^{1 / 2}$, reduction of the error by a factor of 2 necessitates increasing $\mathrm{M}$ by a factor of 4 . Consequently, different "variance reduction" techniques could be employed [2]. Note that in using Method 3, a model for S can depend on 
other Ito processes: for $\mathrm{k}$ processes, a complete set of $\mathrm{N}$ time samples would require $2^{\mathrm{k}(\mathrm{N}+1)}$ computations. Method 2 may require several orders of magnitude of computation more than Method 2.

Methods (1), (2), (3) can also be combined. For example, one can value an American-style derivative with stochastic average interest rate and stochastic average volatility by generating Monte Carlo samples for $\mathrm{r}$ and $\sigma$ as input to a recombining binomial lattice for $\mathrm{f}$. Computational infrastructure is stretched when these three methods are used to value a portfolio of $\mathrm{P}$ derivatives. Consequently, the total amount of computation required for a portfolio is proportional to:

$\mathrm{P}^{*} \mathrm{G}$, for Method 1

$\mathrm{N}^{*} \mathrm{P}$, for Method 2

$\mathrm{N}^{*} \mathrm{M} * \mathrm{P}$, for Method 3

and, in general, the computation time for each method corresponds to

Method $1<$ Method $2<$ Method 3

The problem that we address is concerned with the cost effective computation of the expected value in Equation (1), with respect to the tradeoffs between Methods 1-3 and the above computing topologies. Note that these alternatives are not mutually exclusive, their boundaries are "fuzzy" and they may be combined.

\section{Risk Tradeoffs of Alternative Computing Topologies}

The problem is: given the algorithmic alternatives and parameters $\mathrm{G}, \mathrm{N}, \mathrm{M}, \mathrm{P}$ as defined in Section 2, find a computing topology that minimizes the time and cost required for a valid computation. It is convenient to group the costs into the categories of Opportunity Costs, Infrastructure Costs, and Algorithmic Costs. The first two costs are general and may be applied to any kind of alternative topology problem; Algorithmic Costs are specific to derivative computations. From another perspective, these costs can be used to describe potentially new benefits of changing to an alternative topology: if the business benefit does not outweigh the other costs, then there may be no cost-effective reason to change.

Opportunity Costs. These costs reflect the risks associated with the nature of the routine function of the business. Assessed here are the costs of a late answer, cost of a wrong answer, cost of no answer, and cost of infrastructure breakdown. For example, a fixed income group may require real time evaluation of their entire derivative position 30 minutes before the monthly speech of the Federal Reserve Chairman. If this cannot be done, then there is an opportunity cost.

Infrastructure Cost. These costs reflect the risks associated with maintaining the existing computing infrastructure as well as the additional risks of modifying the infrastructure to a new topology. Assessed here are Client-Server Costs (costs of additional workstations and servers, together with software); Network Costs (costs of network hardware and software); Infrastructure Modification Costs, Runtime Costs; and System Administration Cost. 
The cost and benefit tradeoffs can indicate whether "getting a faster computer" presents a good alternative: the network performance impact is almost as great as the computing processing. For example, purchasing a supercomputer may result in slower performance if the network the supercomputer is on is slow or is saturated with traffic. Figure 1 further illustrates the impact of network performance on computation.

\begin{tabular}{|lrr|}
\multicolumn{1}{l}{} & \multicolumn{1}{c}{ To send 1MB } \\
Ethernet (Network) & No. of float divides \\
requires (sec)... & on 200MHz chip... \\
\hline Regular Ethernet & 0.56 & $112,000,000$ \\
Fast Ethernet & 0.056 & $11,200,000$ \\
OC-3 & 0.051612903 & $10,322,581$ \\
OC-24 & 0.006451613 & $1,290,323$ \\
SP2 & 0.033333333 & $6,666,667$ \\
\hline
\end{tabular}

Tightly Coupled (Parallel Processor Backplane)
\begin{tabular}{|lrr|}
\hline $100 \mathrm{MB} / \mathrm{sec}$ bp & 0.01 & $2,000,000$ \\
$320 \mathrm{MB} / \mathrm{sec}$ & 0.003125 & 625,000 \\
$640 \mathrm{MB} / \mathrm{sec}$ & 0.0015625 & 312,500 \\
$1200 \mathrm{MB} / \mathrm{sec}$ & 0.00078125 & 156,250 \\
\hline
\end{tabular}

Figure 2. Network Speed vs. Computation

This shows, for example, that during the time that one computer is sending another computer 1 Megabyte of data, the other computer could have done over 100 million floating point divides. This latency only gets worse for memory-intensive computation. The derivative evaluation problem is more compute-intensive than memory intensive. On the other hand, some implementations of Method 2 may send large lattices around a network : for $\mathrm{N}=500$, this would amount to about 1 Megabyte.

Algorithmic Costs. These costs reflect the risks associated with maintaining the existing computing algorithm as well as the additional risks of modifying the algorithms to a new topology. Assessed here are the costs of optimizing an algorithm. While many compilers offer one such level of optimization, two other levels of analysis should also be performed. On a macro level, there is a cost-benefit analysis involved in determining the best combination of Methods 1-3. This is essentially the job of the model builder. From a micro perspective, there is a degree of algorithm optimization that is orthogonal to that produced by compiler optimizations. One such optimization is concerned with building a parallel version of the algorithm. The idea here is to implement the algorithm in such a way so that n-processors can solve the problem in (1/nth) the time as one processor.

At this point the tradeoffs between a weakly-coupled parallelism versus a fine-grained parallelism should be addressed.

Method 3 is a problem that can be solved with is weakly-coupled parallelism: for example, Monte Carlo samples can be generated on two different processors, $f$ can be evaluated, and the discounted expected value computed on a third processor. The first two processors are totally 
independent of each other (assuming they both do not generate the same set of "random" samples). Consequently, one can optimally expect a 2:1 speed-up (minus the communication overhead discussed above). Weakly-coupled applications require relatively little effort in creating a parallel speed-up.

Method 2 is a problem that can be solved with fine-grained parallelism It can be shown that each computation along the diagonal of the lattice can be done in parallel. Consequently, an algorithm can be configured that, at time $\mathrm{k}$, computes the values of $\mathrm{S}$ and $\mathrm{f}$ in the $\mathrm{k}+1$ nodes on $\mathrm{k}+1$ processors (see Figure 2).

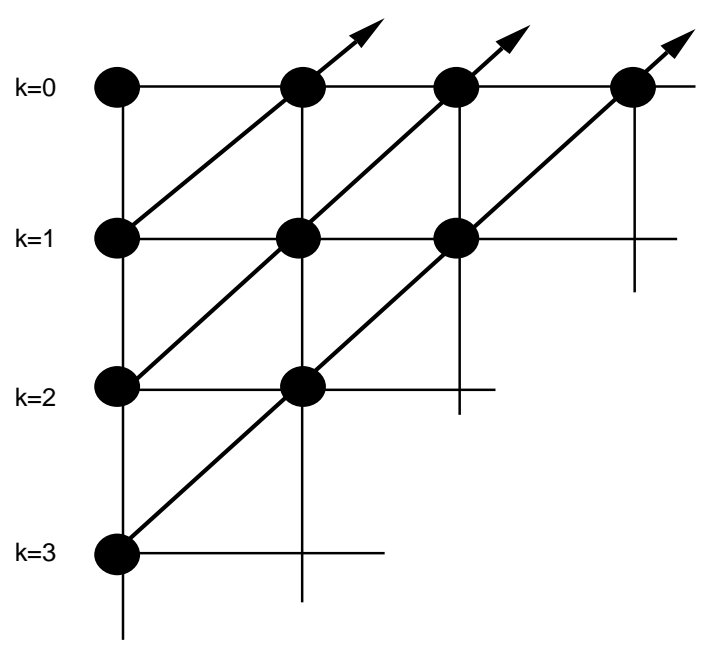

\section{Figure 3. Fine-Grained Parallelism of Recombining Lattice Method}

Consequently, if $\mathrm{N}$ processors are available, instead of performing $(\mathrm{N}+1)(\mathrm{N}+2) / 2$ sequential computations, a fine-grained parallel implementation requires only $(\mathrm{N}+1)$ sequential computations. Fine-grained parallelization usually requires more effort in modifying the algorithm than weakly-coupled parallelization.

Both weakly-coupled and fine-grained parallelization techniques require a topology to support the following parallelization operators:

Broadcast . One processor node sends the same message to other nodes. The simplest broadcast operation is to start running all programs on all nodes.

Scatter. One processor node sends a different message to each node. An example: in Method 3, we can use a scatter operation start running all programs with a different seed to the random number generator.

Gather. Every processor node sends a message to a single member. An example: we gather the Monte Carlo sampled values $f$ for averaging at processor node 0 .

Barrier Synchronization. All processor node must reach the same point before any can proceed. An example: in the fine-grained parallelism of Method 2, computation must synchronize for each diagonal to be completed. 


\section{Evaluating Tradeoffs: An Example}

The following problem, using the most compute-intensive aspects of Method 2 and Method 3, was used as a benchmark in evaluating topology tradeoffs. $\mathrm{S}$ follows an Ito process with constant $\mu$ and $\sigma$, and $f$ is an American-style derivative. We use a recombining lattice to find the expected value of $\mathrm{f}$. Next, we vary the average instantaneous interest rate $\mathrm{r}$ in by taking 1000 Monte Carlo samples. Thus the value of $\mathrm{f}$ is the sample average of 1000 lattice evaluations. The algorithm was implemented to support the weakly-coupled parallelism of Method 3.

We compare the impact of several implementations in Figure 4.

\begin{tabular}{rccc|}
\multicolumn{1}{c}{} & $\begin{array}{c}\text { Time for 1 } \\
\text { Sample }(\mathrm{sec})\end{array}$ & $\begin{array}{c}\text { Time for 1000 } \\
\text { Samples }(\mathrm{sec})\end{array}$ & $\begin{array}{c}\text { No. of } \\
\text { Processors }\end{array}$ \\
\cline { 2 - 4 } Workstation & 5 & 5000 & 1 \\
Faster Workstation & 1 & 1000 & 1 \\
Cluster (PVM) & 3 & crashed & 4 \\
Cluster (PVM/custom) & 3 & $4-103$ & 1000 \\
Cluster-(PVM/SMP) & 0.225 & 225 & 4 \\
Supercomputer & 0.03 & 30 & 40 \\
\hline
\end{tabular}

Figure 4. Benchmark Performance of 6 Computing Topologies

The clusters were implemented under Parallel Virtual Machine, a package that permits the utilization of a heterogeneous network of parallel and serial computers as a single computational resource [7].

The three cluster implementations of the benchmark problem. In the first cluster implementation, the benchmark problem crashed the system. There were too many Monte Carlo requests for the network task scheduler to handle the barrier operations. In the second cluster implementation, the problem was reconfigured to allocate one Monte Carlo sample to each processor. The time required to perform 1000 samples then depended on the latency of the network: it is variable because the network is a shared resource. In the third cluster implementation, the network was a dedicated high-speed backplane (see Figure 2). In this "Symmetric Multi-Processing" implementation, only four processors were allowed to be active at one time.

These results show that the underlying network topology, is the crucial factor in designing cluster computing solutions. Similar results on cluster computing performance are discussed in [8].

\section{An Expert System for Assessing Computing Alternatives}

We have collected several cases that can be used to assess the transition between alternative computing technologies for the optimal computation of Methods 1-3. There are 16 basic cases, 
corresponding to the pairwise transitions between each of the 4 topologies (including the null transition - the alternative of keeping the computing topology the same). Our cases were derived by examining similar transition problems for other compute-intensive applications. Our case profiles include the attributes discussed in Section 3, concerned with opportunity cost, infrastructure cost, and algorithmic cost. As in other case-based reasoning systems, our cases contain typical examples and counter-examples (exceptions). We summarize the conclusions of the typical cases:

\section{Case 1. Workstation to Workstation}

Alternatives provide marginal gain in performance. Alternatives are too expensive. No skills to perform algorithm modification. Algorithm is difficult to parallelize.

\section{Case 2. Workstation to Faster Workstation}

No algorithm modification required. Limited Budget.

\section{Case 3. Workstation to Super Computer}

Algorithm exploits utilization of vectors and vector operations. Budget for the supercomputer is available. Workstations all busy. Bad network infrastructure. Require consistent performance. Skills available to modify algorithm and optimize in FORTRAN. Low modification costs.

\section{Case 4. Workstation to Cluster}

Have many workstations and budget is available to buy more workstations. Other departments will allow limited use of their workstations. Problem cannot be solved with supercomputers.

\section{Case 5. Faster Workstation to Workstation}

Lose of Budget.

Case 6. Faster Workstation to Faster Workstation

Alternatives provide marginal gain in performance. Alternatives too expensive. No skills to do the rehosting. Algorithm is difficult to parallelize.

\section{Case 7. Faster Workstation to Super Computer}

Generally same as workstation to Supercomputer

\section{Case 8. Faster Workstation to Cluster}

Generally same as workstation to Cluster.

\section{Case 9. Supercomputer to Workstation}

Lose of Budget. Performance not good enough to continue justification of Supercomputer. Algorithm is too memory-intensive and too large for the Supercomputer. Staff unable to program in FORTRAN to get maximum Supercomputer performance.

\section{Case 10. Super Computer to Faster Workstation}

Workstations provides equivalent performance at $50 \%$ to $10 \%$ of the price.

\section{Case 11. Super Computer to Cluster}

Lose of Budget. Performance not good enough to continue justification of Supercomputer. There are many workstations available. Algorithm is too memory-intensive and too large for 
Supercomputer. Staff unable to program in FORTRAN to get maximum Supercomputer performance.

\section{Case 12. Super Computer to Super Computer}

Algorithm performance is satisfactory. Algorithm will not work on anything else.

New model upgrade costs are low.

\section{Case 13. Cluster to Workstation}

Solution is having a negative impact on business, primarily due to the saturation of the network. Performance at desktop is being hurt. Everyone is getting a workstation to exploit the computing capability.

\section{Case 14. Cluster to Faster Workstation}

Same as above. Can afford more power per desktop.

\section{Case 15. Cluster to Cluster}

Future model of computing topology. Algorithm performance is satisfactory.

New model upgrade costs are low. New faster network topologies becoming available.

\section{Case 16. Cluster to Supercomputer}

Solution is having a negative impact on business, primarily due to the saturation of the network. Performance at desktop is being hurt. Everyone is getting a workstation to exploit the computing capability. Algorithm exploits utilization of vectors and vector operations. Budget available. Workstations all busy. Bad network infrastructure. Require consistent performance. Skills available to modify algorithm and optimize in FORTRAN. Low modification costs.

The case-based EXpert System to assess Alternative Computing Technologies (EXACT) was implemented in Induce-It [9], a case-based reasoning tool that uses Microsoft Excel spreadsheets as the user interface. Induce-It represents case profiles as values in spreadsheet cells. Case profile attributes are typed and denote either string values (with wildcard matches), ranges, scales, or hierarchies. Each attribute type has its own default attribute similarity metric. Case similarity is defined by combining attribute similarity into a case score.

The EXACT case profile attributes and categories are tabulated in Figure 5.

\begin{tabular}{|lll|}
\hline Category & Case Profile Attribute & Attribute Values \\
\hline Opportunity & Current performance & Poor-Reasonable-Good-Excellent \\
Infrastructure & Budget Available & None-Low-Medium-High \\
Algorithmic & Skills to modify & None-Low-Medium-High \\
Algorithmic & Difficult to parallelize & None-Low-Medium-High \\
Algorithmic & Exploit vectors & None-Low-Medium-High \\
Infrastructure & Workstation Capacity & Not Busy-Somewhat Busy- Very Busy \\
Infrastructure & Network Capacity & Not Busy-Somewhat Busy- Very Busy \\
Infrastructure & Network Infrastucture & Poor-Reasonable-Good-Excellent \\
Algorithmic & FORTRAN Skill Available & None-Low-Medium-High \\
Algorithmic & Memory Intensive & None-Low-Medium-High \\
\hline
\end{tabular}

Figure 5. Case Attributes for EXACT 
These attribute values are implemented as Induce-It scale maps. The case database contains legal values for each attribute or a "don't care" value (Induce-It provides two types of don't care values: one of them is indicated as a "blank" in a case database cell).

In operation, a problem profile representing attributes relating to the case attributes are entered in a spreadsheet region that corresponds to a reference case. EXACT then compares each case to the problem profile, and then ranks all cases by similarity. The case scoring function in EXACT permits a user to weight each attribute. After changing values in the Reference Region or Weights Region, the EXACT spreadsheet is recalculated and the resultant cases are sorted by score. Scores are normalized between 0 and 1: a score of 1 represents a higher match between the case and the reference.

The Induce-It case attribute similarities are stored in a spreadsheet region called the Inductive Database. The entire spreadsheet display can be easily customized: cell regions can be hidden or reformatted.

Here is an example. Suppose that the problem profile indicates the following values:

Problem Profile Attribute
\begin{tabular}{|lc|}
\hline Current performance & Value \\
Budget Available & Poor \\
Skills to modify & Medium \\
Difficult to parallelize & Low \\
Exploit vectors & Medium \\
Workstation Capacity & Medium \\
Network Capacity & Not Busy \\
Network Infrastucture & Somewhat Busy \\
FORTRAN Skill Available & Don't Know or Don't Care \\
Memory Intensive & Low \\
\hline
\end{tabular}

\section{Figure 6. EXACT Problem Profile: An Example}

Here is a display of the case database, scores, weights, reference, and parameters regions. Note that the problem profile is represented as the reference case. All attributes are equally weighted. The ranking in Figure 7 shows that EXACT recommends a cluster topology for this problem.

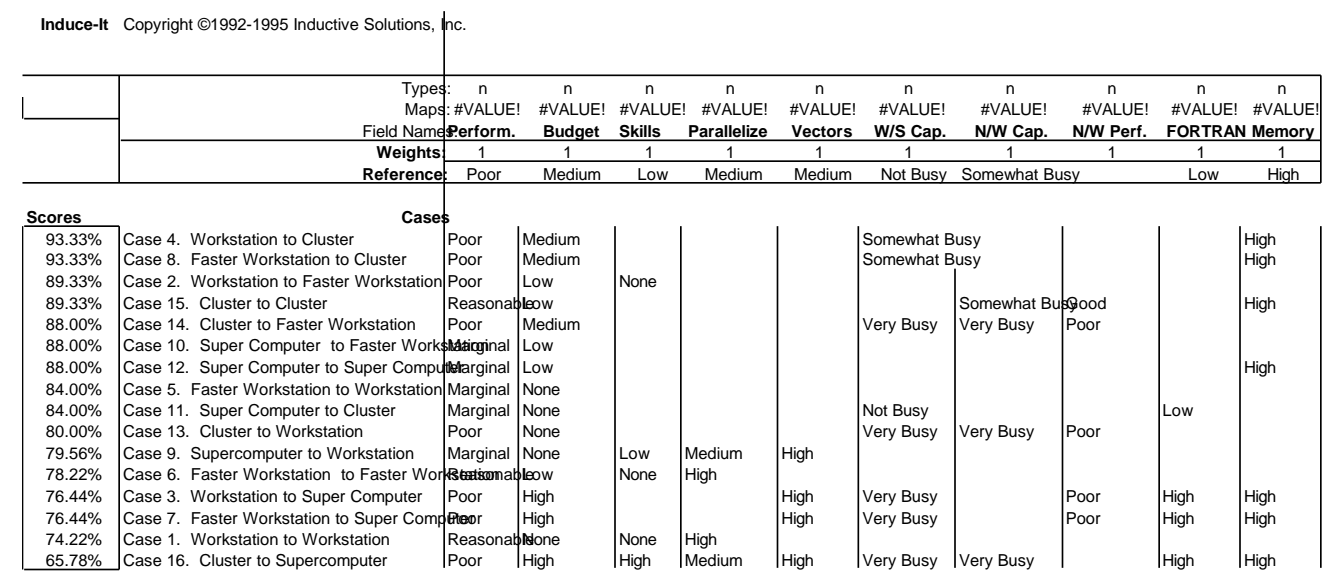

Figure 7. EXACT Problem Profile: Case Rankings 
It seems that as workstation costs decline, the cluster topology becomes more cost effective. However, as seen in the above cases, this alternative is not without problems. A better statement is that as workstation costs and networks improve, the cluster topology will become more cost effective. An important trend that can further help is in the availability of intelligent resource allocation and network utilization systems ([3], [5],[6]).

\section{References}

1. Cagan, L., Carriero, N., and Zenios, S., “A Computer Network Approach to Pricing Mortgage-Backed Securities,” Financial Analysts Journal, March-April 1993.

2. Clewlow, L., and Carverhill, A., “Quicker on the Curves.” Risk, 7(5), May 1994.

3. Huang, C., and McKinley, P., "Communication Issues in Parallel Computing Across ATM Networks," IEEE Parallel \& Distributed Technology, Winter 1994.

4. Ingersoll, J., Theory of Financial Decision Making, Rowman \& Littlefield, 1987.

5. Kaplan, J., and Nelson, M., "A Comparison of Queuing, Cluster, and Distributed Computing Systems," NASA Technical Report TM-109025 (Revision 1), June 1994.

6. Lirov, Y., et al, "Intelligent Infrastructure for the Distributed Front Office," in Artificial Intelligence in the Capital Markets, ed. by R.S. Freedman, R. Klein. \& J. Lederman, Probus, 1995.

[7] Beguelin, A., et al, A Users' Guide to PVM Parallel Virtual Machine, Oak Ridge National Laboratory, U.S. Department of Energy Contract, DE-AC-05-84OR21400.

[8] Anderson, T., et al, “A Case for NOW (Networks of Workstations)," Report for Advanced Research Projects Agency, Contract N00600-93C-2481.

[9] Induce-It User Manual. Inductive Solutions, Inc., 1992-1995. 